\title{
Fusarium verticillioides Induces Maize-Derived Ethylene to Promote Virulence by Engaging Fungal G-Protein Signaling
}

\author{
Yong-Soon Park, ${ }^{1}$ Eli J. Borrego, ${ }^{1}$ Xiquan Gao, ${ }^{1}$ Shawn A. Christensen, ${ }^{1,2}$ Eric Schmelz, ${ }^{2}$ \\ Alessandra Lanubile, ${ }^{3}$ Dillon A. Drab, ${ }^{1}$ Will Cody, ${ }^{1}$ Huijuan Yan, ${ }^{1}$ Won-Bo Shim, ${ }^{1}$ and \\ Michael V. Kolomiets ${ }^{1, \dagger}$ \\ ${ }^{1}$ Department of Plant Pathology and Microbiology, Texas A\&M University, College Station, TX 77843-2132, U.S.A. \\ ${ }^{2}$ Chemistry Unit, Center of Medical, Agricultural, and Veterinary Entomology, United States Department of Agriculture, \\ Gainesville, FL 32608, U.S.A. \\ ${ }^{3}$ Department of Sustainable Crop Production, Università Cattolica del Sacro Cuore, Piacenza, Italy
}

Accepted 22 June 2021.

Seed maceration and contamination with mycotoxin fumonisin inflicted by Fusarium verticillioides is a major disease concern for maize producers worldwide. Meta-analyses of

Yong-Soon Park and Eli J. Borrego contributed equally to this work.

Current address for Yong-Soon Park: Biotechnology Research Institute, College of Natural Sciences, Chungbuk National University, Cheongju 28644, South Korea.

Current address for Eli J. Borrego: Thomas H. Gosnell School of Life Sciences, Rochester Institute of Technology, Rochester, NY 14623, U.S.A.

Current address for Xiquan Gao: State Key Laboratory of Crop Genetics and Germplasm Enhancement, College of Agriculture, Nanjing Agricultural University, Nanjing, China

Current address for Eric Schmelz: Section of Cell and Developmental Biology, University of California at San Diego, La Jolla, CA, U.S.A.

Current address for Will Cody: Department of Chemical Engineering, Stanford University, Stanford, CA, U.S.A.

${ }^{\dagger}$ Corresponding author: M. V. Kolomiets; kolomiets@tamu.edu

Funding: This work was supported by the Texas AgriLIFE Research Monocot Improvement Program, National Science Foundation grants IOB0544428, IOS-0925561, and IOS-0544428, National Research Initiative Competitive Grants Program grant 2007-35319-18334, and a United States Department of Agriculture National Institute of Food and Agriculture Plant Biotic-Interactions Program grant 2017-67013-26524 from the United States Department of Agriculture National Institute of Food and Agriculture. It was also supported by the National Science Foundation of China (grant numbers 31471508 and 31671702) and Jiangsu Collaborative Innovation Center for Modern Crop Production (JCIC-MCP) to X. Gao. In addition, this research was supported by the Basic Science Research Program through the National Research Foundation of Korea (NRF) funded by the Ministry of Education (NRF-2018R1D1A3B07049986) to Y.-S. Park. We thank the Texas A\&M University Department of Plant Pathology and Microbiology Bioenvironmental Undergraduate Research Scholars (BURS) program for the support of D. A. Drab.

*The $\boldsymbol{e}$-Xtra stands for "electronic extra" and indicates there are supplementary materials published online.

The author(s) declare no conflict of interest.

(c) (1) $(\Theta)$ Copyright $(92021$ The Author(s). This is an open access article distributed under the CC BY-NC-ND 4.0 International license. quantitative trait loci for Fusarium ear rot resistance uncovered several ethylene (ET) biosynthesis and signaling genes within them, implicating ET in maize interactions with $F$. verticillioides. We tested this hypothesis using maize knockout mutants of the 1-aminocyclopropane-1-carboxylate (ACC) synthases ZmACS2 and ZmACS6. Infected wild-type seed emitted five-fold higher ET levels compared with controls, whereas ET was abolished in the acs 2 and acs6 single and double mutants. The mutants supported reduced fungal biomass, conidia, and fumonisin content. Normal susceptibility was restored in the acs6 mutant with exogenous treatment of ET precursor ACC. Subsequently, we showed that fungal G-protein signaling is required for virulence via induction of maize-produced ET. $F$. verticillioides $\mathbf{G}_{\beta}$ subunit and two regulators of $\mathbf{G}$-protein signaling mutants displayed reduced seed colonization and decreased ET levels. These defects were rescued by exogenous application of ACC. We concluded that pathogen-induced ET facilitates $F$. verticillioides colonization of seed, and, in turn, host ET production is manipulated via G-protein signaling of $F$. verticillioides to facilitate pathogenesis.

Keywords: ACC synthase, ethylene, F. verticillioides, G-protein signaling, maize

Fusarium and Aspergillus spp. are the most common mycotoxin-producing fungi that contaminate a large number of crops at pre- and postharvest stages (Munkvold 2003). In the U.S. and Europe, major economic losses associated with mycotoxins are from fumonisin (produced primarily by Fusarium verticillioides), trichothecene and zearalenone (produced by Fusarium spp.), and aflatoxin (produced by Aspergillus spp.) contamination of commodities. Unfortunately, conventional approaches have had limited success in reducing mycotoxin contamination (Munkvold 2003). One ideal solution is to minimize contamination by improving the genetic resistance of crops. However, despite significant investment in breeding programs, adequate levels of resistance have not been achieved, primarily due to the absence of major resistance genes against any of these pathogens (Lanubile et al. 2017; Munkvold 2003). One promising approach to enhance conventional breeding efforts is to identify the genes governing resistance (or susceptibility). The most efficient means of identifying these candidate genes is the identification of quantitative trait loci (QTL) by analyzing genome-wide association studies of the 
populations derived from parents that contrast in their resistance against Fusarium ear rot and fumonisin contamination levels (Lanubile et al. 2017). When association of these candidate genes to the traits is characterized, beneficial crop alleles can be utilized to develop genetic markers or genetic engineering approaches to control pathogen infection and mycotoxin production.

An important pathway proposed in defense against $F$. verticillioides is the ethylene (ET) $\left(\mathrm{C}_{2} \mathrm{H}_{4}\right)$ signaling pathway (Lanubile et al. 2017). While there is no experimental evidence to support this hypothesis, this notion is reasonable, due to the fact that phytohormone levels are altered following pathogen attacks and this results in massive transcriptional reprogramming in a pathogen-specific defense response (Kunkel and Brooks 2002). Among the many molecules known to be involved in plant defense, ET is the only phytohormone that exists in a gaseous state and for which receptors have been identified. ET activity and biosynthesis has been extensively studied in plants since the 1930s (Gane 1934). In higher plants, ET is synthesized from methionine via $S$-adenosyl-L-methionine (SAM) by SAM synthetase. SAM is subsequently converted to 1-aminocyclopropane-1-carboxylate (ACC) by ACC synthase (ACS) (SAM methylthioadenosine-lyase) and, finally, to ET by ACC oxidase (ACO) (Kende 1993; Yang and Hoffman 1984). ET regulates diverse physiological processes (Johnson and Ecker 1998; Kende 1993) and defense responses against abiotic and biotic stress factors (Adie et al. 2007; Broekaert et al. 2006; Guo and Ecker 2004; Kendrick and Chang 2008; Lin et al. 2009). Many reports illustrate how ET regulates plant defense response to diverse pathogens in combination with other plant-derived hormones (Broekaert et al. 2006; Ellis and Turner 2001; Glazebrook et al. 2003; Penninckx et al. 1998).

While the majority of plant-pathogen interaction studies have revealed defense-related roles for ET, there are other reports demonstrating how ET contributes toward increased pathogen virulence and plant host susceptibility (Barkai-Golan et al. 1989; El-Kazzaz et al. 1983; Wang et al. 2017). Despite the crucial role of ET in a variety of physiological processes in plants and microbes, there is little genetic evidence to support the function of host-derived ET in maize pathogenesis, namely on the ability of fungal pathogens to colonize, reproduce, and synthesize mycotoxins. We recognize that there are many contradicting results showing ET as a defense molecule or a susceptibility factor in plant-pathogen studies (Bari and Jones 2009; Cristescu et al. 2002, Ma and Ma 2016). Despite numerous studies on mechanism of ET biosynthesis and signaling, little is known about the particular gene family members responsible for ET biosynthesis, especially in the seed tissue. Our recent study demonstrated that ET emitted by maize seed facilitates Aspergillus flavus colonization but not mycotoxin production, indicating that maize seed-derived ET modulates plant susceptibility and fungal growth (Wang et al. 2017).

Previously, we demonstrated that two regulators of G-protein signaling in $F$. verticillioides, FLBA1 and FLBA2, are required for the pathogen to effectively colonize seed, reproduce, and synthesize fumonisin $\mathrm{B}_{1}\left(\mathrm{FB}_{1}\right)$ (Mukherjee et al. 2011). Furthermore, strains disrupted in these genes were unable to induce ET biosynthesis during seed colonization (Mukherjee et al. 2011). These results prompted us to hypothesize that the G-protein signaling pathway is responsible for pathogen-host communication that directly or indirectly induces ET biosynthesis in maize seed. To broaden our knowledge of ET in regulating the interaction between maize seed and fungi, we investigated the effect of maize ET biosynthesis and resistance against $F$. verticillioides. In this study, we provide evidence that host-derived ET is necessary for fungal colonization, reproduction, and mycotoxin production and that host ET biosynthesis may be manipulated by
F. verticillioides via fungal G-protein signaling to subsequently promote its virulence.

\section{RESULTS}

\section{The maize genome encodes five $A C S$ and $13 A C O$ gene family members.}

Recent advances in single molecule sequencing technology have improved the quality of maize reference genome annotation (Jiao et al. 2017) that resulted in approximately 250-fold greater contiguity compared with previous short-read technologies. This development warranted a re-analysis of the genes associated with ET biosynthesis, perception, and signaling. Compared with the 11-member Arabidopsis ACS gene family (Yamagami et al. 2003), the maize genome encodes five ACS proteins, ZmACS1, ZmACS2, ZmACS3, ZmACS6, and ZmACS7 (Fig. 1A). These proteins share between 34 to $94 \%$ amino acid sequence identity among themselves and against their Arabidopsis orthologs and cluster into four distinct groups (Supplementary Fig. S1A). ZmACS1 shares the highest homology with AtACS7, while ZmACS2 and ZmACS7 share the greatest similarity with each other and group with AtACS1, AtACS2, and AtACS6. ZmACS6 is orthologous to five Arabidopsis protein family members (AtACS4, AtACS5, AtACS8, AtACS9 and AtACS11) from one phylogenetic clade. ZmACS3 shares the greatest homology with the non-ACC producing AtACS10/12 and, therefore, is not likely responsible for ET biosynthesis.

In the process of identifying the maize $A C O$ gene family members, several $A C O$ candidates were annotated as benzoxazinoid, flavonoid, and gibberellic acid biosynthesis genes and formed distinct clusters. These genes were excluded from further analyses, as they likely belong to other 2-oxoglutarate oxygenase subfamilies. In the future, these discrepancies in annotations will necessitate a comprehensive biochemical analysis of recombinant proteins for validating the true maize (and Arabidopsis) ACO family members. Following the most conservative approach using sequence similarity with the $A C O$ gene family in Arabidopsis (Ruduś et al. 2013), we concluded that the maize genome harbors $13 A C O$ gene family members (Fig. 1B). These maize genes cluster into four distinct groups and share 40.1 to $66.4 \%$ amino acid sequence similarity with Arabidopsis ACO proteins (Supplementary Fig. S1B). The clade containing ZmACO1 has two members and shares homology with a single AtACO1 protein. $\mathrm{ZmACO} 2 \mathrm{a}, \mathrm{ZmACO} 2 \mathrm{~b}$, and $\mathrm{ZmACO} 2 \mathrm{c}$ cluster with the orthologs, AtACO2, AtACO3, and AtACO4. AtACO5 shared a clade with ZmACO3a, ZmACO3b, $\mathrm{ZmACO} 3 \mathrm{c}$ and with the nearby group composed of five tandemly duplicated near-isogenic paralogs, ZmACO4a, ZmACO4b, ZmA$\mathrm{CO} 4 \mathrm{c}, \mathrm{ZmACO} 4 \mathrm{~d}$, and $\mathrm{ZmACO} 4 \mathrm{e}$.

To gain further insight into ET pathway components, we also examined the genes involved in ET perception and signaling in the updated V4 version of the B73 maize genome (Cannon et al. 2011). The maize ET receptor gene family contained eight members that clustered into three groups (Supplementary Fig. S2), while the CTR1 gene family contained two CTR1 paralogs and a closely related $C R G 1$ ortholog (Supplementary Fig. S3). There appears to be only one maize EIN2 with high homology to AtEIN2 (Supplementary Fig. S4) and nine EIN3/EIL1-like gene family members (Supplementary Fig. S5).

To understand if there were any known relationships in maize- $F$. verticillioides interactions and ET, the genes associated with ET biosynthesis, perception, and signaling were placed on a chromosomal map of previously published QTL for Fusarium ear rot resistance (Fig. 1C; Supplementary Table S1). The ET biosynthesis gene ZmACS2 was located in a QTL identified on chromosome 2, found in a biparental population derived from the cross between resistant (CO441) and susceptible (CO354) maize lines (Maschietto et al. 2017). CTRIa and EILAa were found in a 
QTL identified on chromosome 5 derived from NCB (NC300 X B104) and GEFR (FE400 X FR1064) populations (Robertson Hoyt et al. 2006). ACO2a, ACOla, and EILIb were found in the QTL on chromosome 5, 8 and 9, respectively (Maschietto et al. 2017). The most interesting finding was that the entire ACO4 clade, consisting of five members, is found on a QTL identified in chromosome 10 in a cross between a resistant (87-1) and a susceptible (Zong3) line (Ding et al. 2008) (Fig. 1C). Of all the ET-related maize genes identified, only ZmACS2 and ZmACS6 have knockout mutants available at the near-isogenic stage (back cross 7) (Young et al. 2004), and thus, we focused our subsequent genetic analysis on these two genes.

\section{Infection by $\boldsymbol{F}$. verticillioides induces}

ET biosynthesis genes in maize seed.

To test the involvement of maize ET in response to seed infection by $F$. verticillioides, transcript levels of ZmACS2, ZmACS6, and ZmACO3/4 genes (primers used are capable of amplification of transcripts for all of the following genes, $A C O 3 a, A C O 3 b, A C O 3 c, A C O 4 a, A C O 4 b, A C O 4 c, A C O 4 d$, and $A C O 4 e$ ) were monitored at $0,12,24,48$, and $96 \mathrm{~h}$ postinoculation (hpi) (Fig. 2A). All tested genes were induced upon inoculation with $F$. verticillioides when compared with the control treatment (Fig. 2A). The ZmACS2 and ZmACS6 transcripts were induced between 24 to $96 \mathrm{hpi}$. ZmACO3/4 gene members were also induced (Fig. 2A). Notably, the transcripts of the two $Z m A C S$ genes were detected in $F$. verticillioides-inoculated seed at a relatively early timepoint when compared with the transcript of $Z m A C O 3 / 4$, which is in agreement with the sequential enzymatic order involved in ET biosynthesis. Taken together, these results showed that ET biosynthetic genes were induced during $F$. verticillioides infection of seed and suggested that ET production is associated with disease progression.

\section{Maize acs 2 and acs 6 mutants are appropriate genetic tools for exploring the role of ET \\ in maize seed- $\boldsymbol{F}$. verticillioides interaction.}

To understand the dynamics of ET emissions during maize seed infection by $F$. verticillioides, a timecourse experiment was performed in which ET was quantified at 1, 2, 4, 5, 6, 7, and 8 days postinoculation (dpi) from maize seeds infected with $F$. verticillioides. ET levels increased slightly from 1 to 2 and 4 dpi. There was a dramatic increase in ET production between 4 and 5 dpi and continued increase in ET was observed until the maximum production was reached at $7 \mathrm{dpi}$ (Fig. 2B). ET emission in dry seed before inoculation was below detection limit in our study (data not shown).

To explore the role of ZmACS2 and ZmACS6 during $F$. verticillioides seed infection, we took advantage of the previously reported acs 2 and acs6 mutants (Young et al. 2004). The suitability of these mutants was first validated in terms of ET production. While acs2-1 and acs6-1 (herein referred to as acs2 and acs6) Mutator-transposon insertional alleles showed a dramatic reduction in foliar ET (approximately 40 and 90\%, respectively) under basal conditions (Young et al. 2004), the same mutants emitted strikingly greater levels of ET (approximately
A

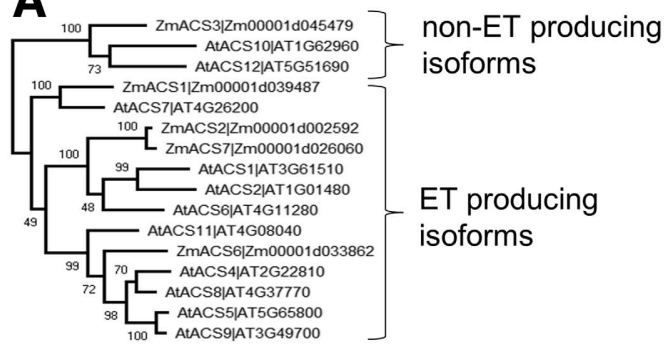

025

B

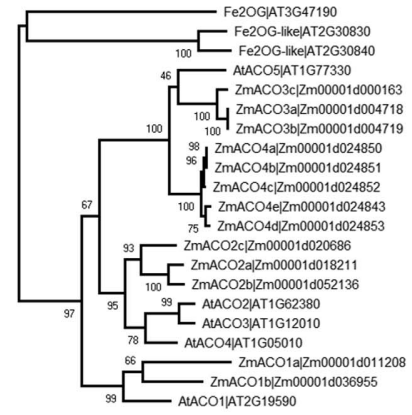

\section{C}

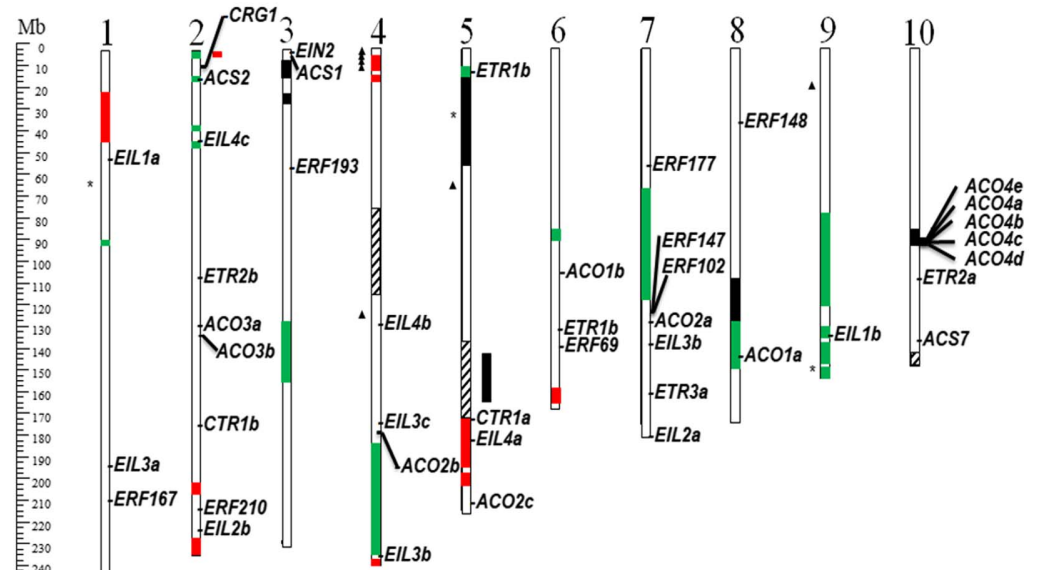

Modified from Lanubile et al. 2017

- ETR1a

- Robertson-Hoyt et al. 2006

- Ding et al. 2008

Mma Chen et al. 2012

- Maschietto et al. 2017

- Samayoa et al. 2019

Fig. 1. The maize 1-aminocyclopropane-1-carboxylate (ACC) synthase (ACS) and ACC oxidase gene family members and position of ethylene (ET)-related genes on a quantitative trait loci (QTL) map of maize Fusarium ear rot resistance. A, The evolutionary history was inferred by using the maximum likelihood method based on the JTT matrix-based model (Jones et al. 1992). The tree with the highest log likelihood (-8,304.09) is shown. The percentage of trees in which the associated taxa clustered together is shown next to the branches. Initial trees for the heuristic search were obtained automatically by applying neighbor-joining and BioNJ algorithms to a matrix of pairwise distances, estimated using a JTT model, and then, selecting the topology with superior log likelihood value. The tree is drawn to scale, with branch lengths measured in the number of substitutions per site. The analysis involved 21 amino acid sequences. There were a total of 420 positions in the final dataset. Evolutionary analyses were conducted in MEGA X (Kumar et al. 2018). B, The tree with the highest log likelihood $(-8,304.09)$ is shown. The percentage of trees in which the associated taxa clustered together is shown next to the branches. Initial trees for the heuristic search were obtained automatically by applying neighbor-joining and BioNJ algorithms to a matrix of pairwise distances, estimated using a JTT model, and then, selecting the topology with superior log likelihood value. The tree is drawn to scale, with branch lengths measured in the number of substitutions per site. The analysis involved 21 amino acid sequences. There were a total of 420 positions in the final dataset. Evolutionary analyses were conducted in MEGA X (Kumar et al. 2018). C, Position of ET-related genes within previously identified QTL for Fusarium ear rot resistance. Modified from Lanubile et al. (2017). 
50 and $600 \%$, respectively) during seed infection with A. flavus (Wang et al. 2017). Because of this tissue- and pathogendependent effect on ET emissions, these knockout alleles were analyzed by measuring ET emission in response to $F$. verticillioides infection. Seeds of the acs mutant lines were inoculated with $F$. verticillioides spores and ET production was monitored at 1, 2, 4, and 7 dpi. At $7 \mathrm{dpi}$, wild-type (WT) maize seeds infected with $F$. verticillioides showed a six- to around 12-fold increase in ET levels when compared with earlier timepoints and noninoculated controls (Fig. 2C). In agreement with the contribution of both ZmACS2 and ZmACS6 to ET synthesis in this pathosystem, headspace from neither acs2, acs6, nor acs 2 acs6 accumulated WT levels of ET. This outcome suggested that the $\operatorname{acs} 2$ and acs 6 knockout mutants are unable to produce pathogen-induced ET production and are thus suitable for assessing the function of ET in maize interaction with $F$. verticillioides.

\section{ZmACS2 and ZmACS6 are required to support normal} colonization, sporulation, and $\mathrm{FB}_{1}$ production

in $\boldsymbol{F}$. verticillioides.

To study the specific role of the host-derived ET in the interaction between maize seeds with $F$. verticillioides, acs 2 and acs 6 single mutants and acs 2 acs 6 double mutants were tested for their ability to support fungal colonization, sporulation, and $\mathrm{FB}_{1}$ production compared with WT. Kernel infection bioassays showed that all three acs mutants displayed substantially reduced colonization by $F$. verticillioides, as evidenced by a decrease in visible fungal growth (Fig. 2D) and reduction in fungal biomass measured by the fungal-specific membrane lipid ergosterol (Fig. 2E). Reduced fungal colonization was accompanied by a 1.5- to twofold reduction in the number of microconidia produced on the acs mutant seeds as compared with the WT (Fig. 2F). Consistent with the substantial reduction in fungal growth and sporulation, the fungus produces significantly less $\mathrm{FB}_{1}$ while growing on the acs mutant seeds (Fig. 2G). Collectively, these findings provide evidence that the ZmACS2 and ZmACS6 genes are required for normal disease progression in seed.

\section{Exogenous ACC treatment restores susceptibility of acs 6 mutants in a dose-dependent manner.}

To provide pharmacological evidence to the question of whether restoring ACC production in the acs mutants rescues the susceptibility phenotype to the levels observed in the WT seed, acs6 mutants were chemically complemented by adding exogenous ACC every other day following fungal inoculation. ACC treatment resulted in the restoration of normal disease severity in the acs6 mutant, as evidenced by visually increased seed colonization (Fig. 3A) and increased conidiation (Fig. 3B). A dose of $1 \mathrm{mM}$ ACC proved to be the most effective concentration for complementing acs6. These data strongly support our
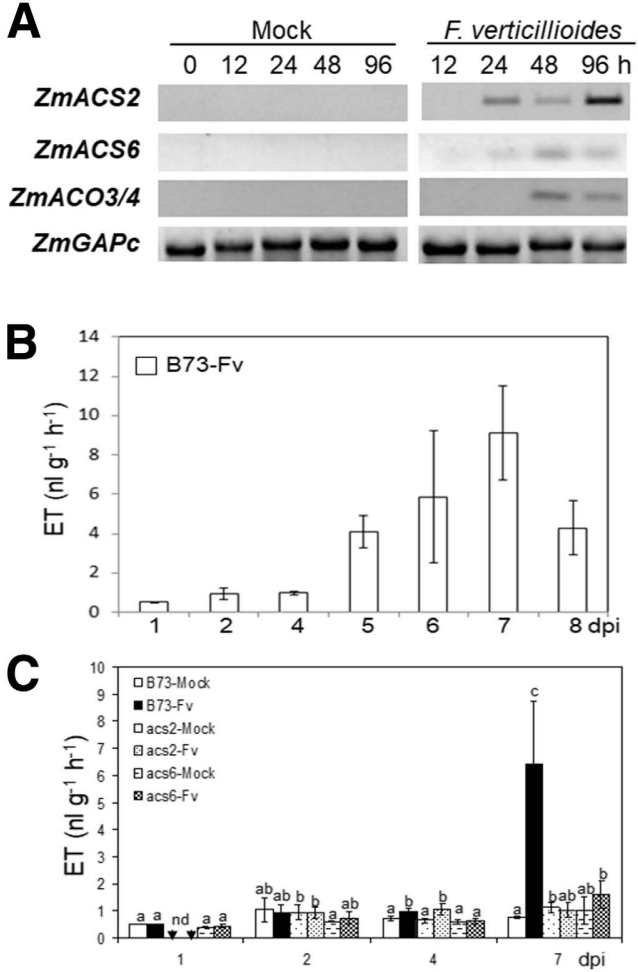
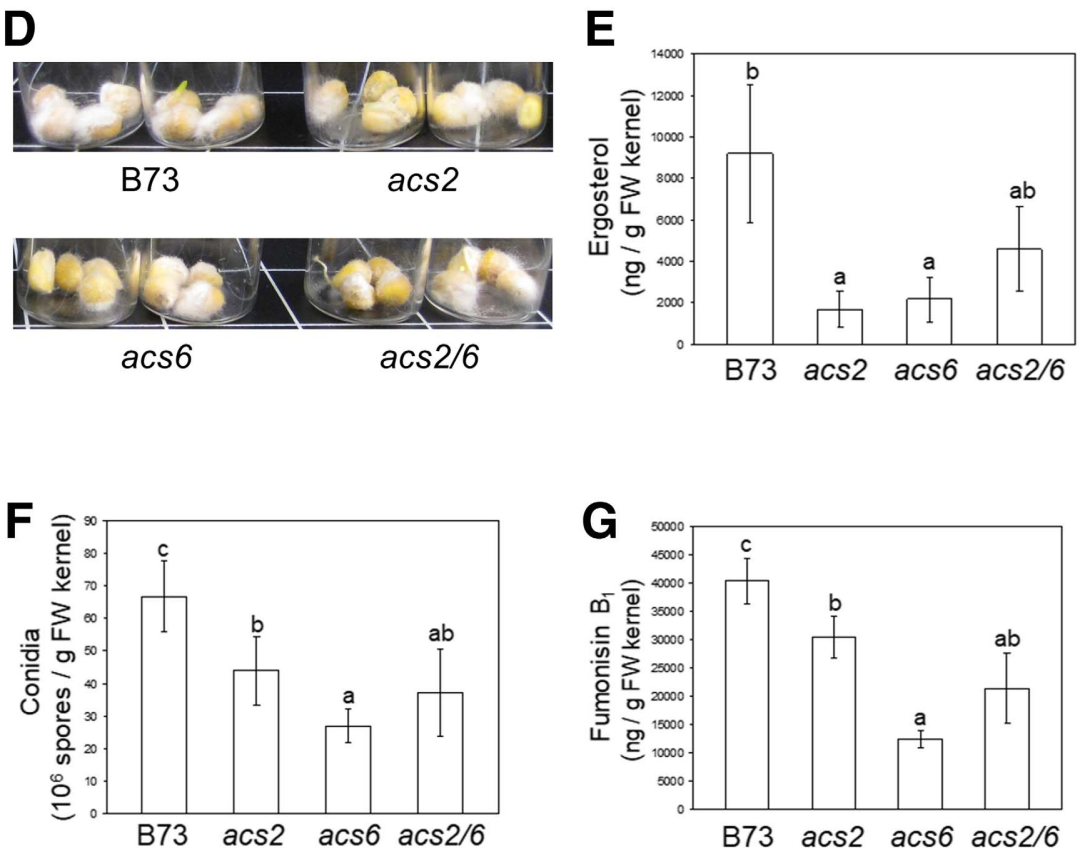

Fig. 2. ZmACS2 and ZmACS6 are induced during Fusarium verticillioides infection and are required for ethylene (ET) production and susceptibility. A, Genes encoding ET biosynthesis enzymes, 1-aminocyclopropane-1-carboxylate (ACC) synthase (ZmACS2 and ZmACS6), and ACC oxidase (ZmACO3 and four clade members) were induced in maize seed in response to $F$. verticillioides infection. B73 inbred line seeds were inoculated with $F$. verticillioides or were mock-inoculated and were incubated and harvested at $0,12,24,48$, and $96 \mathrm{~h}$ postinoculation. Expression of housekeeping gene ZmGAPc was used as an internal standard in this experiment. B, ET was quantified at 1, 2, 4, 5, 6, 7, and 8 days postinoculation (dpi) in the B73 kernels in response to $F$. verticillioides. The vials containing infected kernels were tightly sealed for $3 \mathrm{~h}$ prior to withdrawal of headspace gases to allow detection of ET accumulation by gas chromatography. C, ET production by B73 and near-isogenic acs mutants infected with $F$. verticillioides. ET was measured in B73, acs2, and acs6 kernels at 1,2, 4, and 7 dpi with $F$. verticillioides suspension or mock control. Letters above the bars indicate a significant difference $(P=0.05)$ between the host genotypes within the same timepoint. nd $=$ not detectable. $\mathbf{D}$ through $\mathbf{G}, F$. verticillioides colonization, conidiation, and fumonisin $\mathrm{B}_{1}$ production when grown on the wild-type (B73) and acs mutant kernels. D, Phenotypes of B73, acs 2 , acs 6 and acs $2 / 6$ kernels infected by F. verticillioides at 7 dpi. E, Ergosterol, a fungal-specific membrane lipid, was measured using high performance liquid chromatography (HPLC). F, Conidiation of $F$. verticillioides grown on the $\mathrm{B} 73$ and the acs mutants was measured by hemocytometer. G, Fumonisin $\mathrm{B}_{1}\left(\mathrm{FB}_{1}\right)$ was quantified using HPLC. Bars represent means of four or five biological replicates \pm standard deviation. Letters above the bars indicate significant difference $(P=0.05)$ between genotypes by analysis of variance. 
hypothesis that ET is required for the normal susceptibility of maize seeds to $F$. verticillioides.

\section{Maize seed, not $F$. verticillioides, is responsible for ET production.}

To elucidate the origin of induced ET during $F$. verticillioides seed infection, non-viable seeds were inoculated with $F$. verticillioides and were tested for ET production at 4 and $7 \mathrm{dpi}$. Similar to $F$. verticillioides inoculation on viable kernels, fungal colonization (Fig. 4A) and conidiation (Fig. 4C) increased at 4 and $7 \mathrm{dpi}$ in non-viable kernels. In sharp contrast to viable seeds, no ET emission was detected from infected non-viable kernels (Fig. 4B). These data demonstrated that the seed and not the fungus is the primary source of ET upon colonization by $F$. verticillioides. However, fungal-derived ET cannot be completely ruled out, since the level of production could be below detection limits of the analysis.

\section{$F$. verticillioides $G$ protein $\beta$ subunit $G B B 1$ gene is required for $\mathrm{ET}$ induction and pathogenicity.}

$F$. verticillioides virulence was significantly impaired on the acs mutants (Fig. 2), which led us to hypothesize that $F$. verticillioides manipulates ET biosynthesis via yet-to-be-determined fungal signaling pathways during the course of maize kernel colonization. To test this hypothesis, we studied how $F$. verticillioides $\mathrm{G}$ protein $\beta$ subunit knockout mutant strain $\Delta g b b 1$ (Sagaram and Shim 2007) affects ET biosynthesis in seed. The $\Delta g b b 1$ mutant strain displayed significantly reduced colonization, as evidenced by visually less fungal mycelia growth on the kernel (Fig. 5A) and lower ergosterol content in infected kernels (Fig. 5B). Consistent with this reduced colonization, $\Delta g b b 1$ produced significantly less conidia (Fig. 5B) and $\mathrm{FB}_{1}$ compared with the WT strain (Fig. 5C). In agreement with the hypothesis, the $\Delta g b b l$ strain failed to induce ET at all timepoints tested, while the WT was capable of inducing ET biosynthesis in seed (Fig. 5E). These data suggest that $G B B 1$ and a functional G-protein signaling pathway are required to induce ET production during seed infection by $F$. verticillioides. This result supported the findings from our previous study showing that another G-protein signaling mutant, $\triangle f b A 1$, also failed to induce detectable levels of ET (Mukherjee et al. 2011).

To test whether the inability of G-protein mutants of $F$. verticillioides to induce ET can explain seed colonizing deficiencies in these fungal strains, exogenous ACC treatment was used to rescue virulence of $\triangle g b b l$ and $\triangle f b A l$ strains. Our data showed that both 0.1 and $1 \mathrm{mM} \mathrm{ACC}$ treatment led to increased colonization and conidiation in $\Delta g b b 1$ strain to about $60 \%$ of the WT levels (Fig. 6A and B). Notably, fungal growth and conidiation in the $\triangle f b A l$ mutant were increased to the levels corresponding to the WT strain in a dose-dependent manner, with $1 \mathrm{mM} \mathrm{ACC}$ completely reversing the mutant virulence phenotypes (Fig. 6). These data provide evidence that one potential mechanistic explanation for reduced virulence observed in $F$. verticillioides G-protein mutants is their inability to induce ET biosynthesis in their host as one of the pathogenicity strategies utilized by this fungus. During the search for fungal G protein-dependent signals responsible for regulating ET, a metabolite was discovered
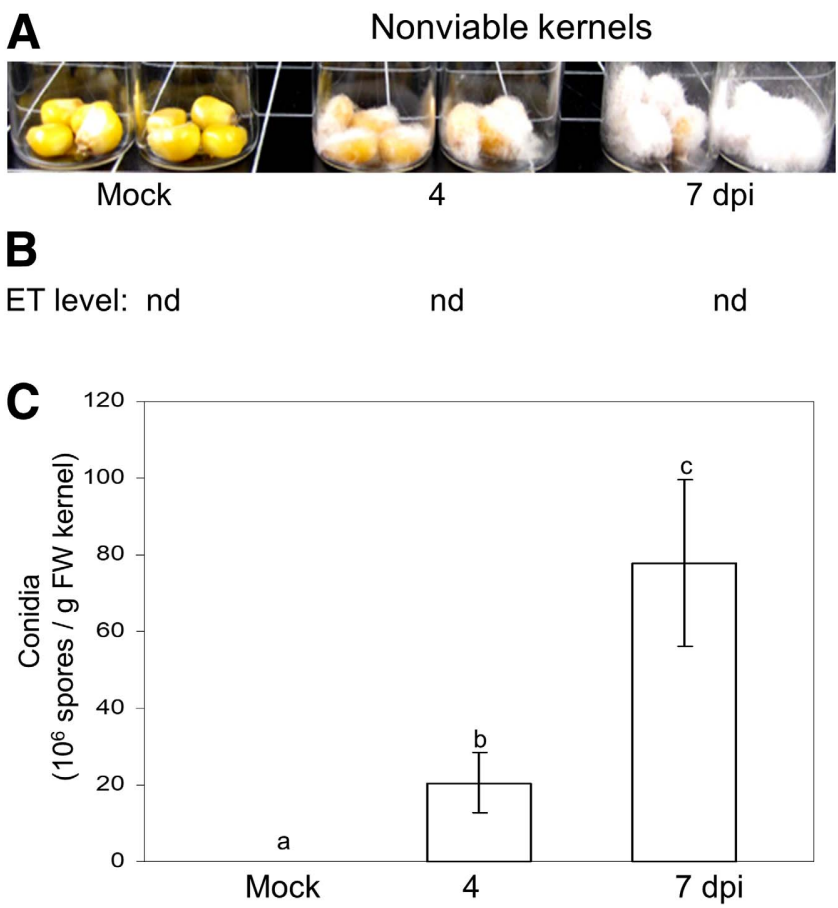

Fig. 4. The maize seed is responsible for ethylene (ET) biosynthesis during Fusarium verticillioides infection. A, B73 kernels were killed by autoclaving for $5 \mathrm{~min}$. The non-viable kernels were inoculated with either control or fungal suspension. B, ET was quantified at 4 and 7 days postinoculation (dpi). C, Conidia were counted at 4 and 7 days after inoculation on non-viable kernels. Bars represent means of 4-5 biological replicates \pm standard deviation. Letters above the bars indicate significant difference $(P=0.05)$ between genotypes by analysis of variance.
A

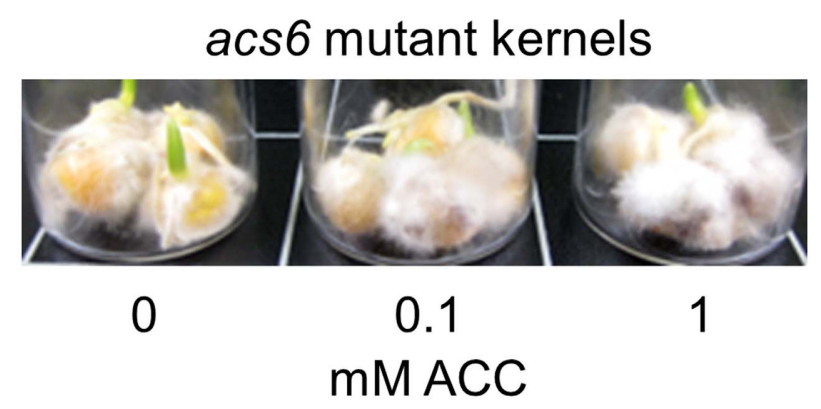

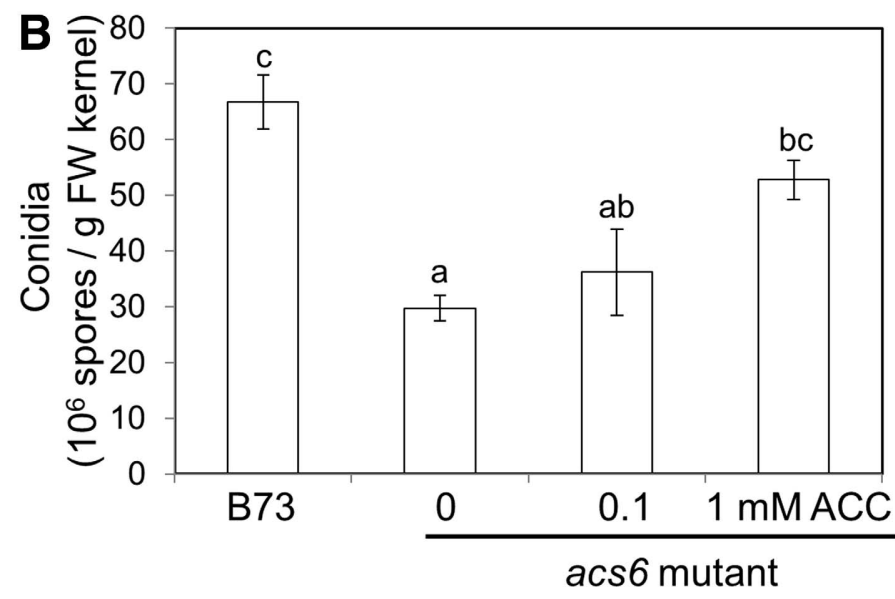

Fig. 3. Exogenous treatment with the ethylene (ET) precursor 1-aminocyclopropane-1-carboxylate (ACC) rescues susceptibility of acs6 mutants to Fusarium verticillioides. A, Colonization and $\mathbf{B}$, conidiation infected with $F$. verticillioides wild-type strain were performed by adding either control or ACC $(0.1$ and $1 \mathrm{mM})$ for 9 days postinoculation at $27^{\circ} \mathrm{C}$. Bars represent means of four to five biological replicates \pm standard error. Letters above the bars indicate significant difference $(P=0.05)$ between genotypes by analysis of variance. 
that correlates with fungal virulence and agrees with a mass fragmentation pattern of a molecule similar to oxylipin 9-oxooctadecatrienoic acid (Supplementary Fig. S6). However, the exact identity of this molecule will need to be further investigated by nuclear magnetic resonance (NMR) analyses as part of our future studies.

\section{DISCUSSION}

Despite years of research into the role of ET in plant defense against pathogens, nearly all reports focused on leaf tissue and in dicot species. The relevance of ET to resistance against seedcolonizing pathogens is less understood. Here, we provided genetic and pharmacological evidence for the role of ET as a susceptibility factor in maize $-F$. verticillioides pathosystem and showed that host-derived ET is necessary for supporting pathogenesis, including fungal colonization, reproduction, and mycotoxin biosynthesis. Two major conclusions are drawn based on our findings. First, seed-derived ET is required for normal fungal virulence, including the ability of the fungus to colonize seed, produce spores, and synthesize $\mathrm{FB}_{1}$. Second, $F$. verticillioides G-protein signaling is a pathway through which, directly or indirectly, the fungus can induce host-derived ET production.

ET biosynthesis gene transcripts accumulated in response to infection with $F$. verticillioides as early as $1 \mathrm{dpi}$ (Fig. 2A). This is in agreement with previous studies showing induction of ET biosynthetic genes in response to infection by diverse Fusarium spp. (Gottwald et al. 2012; Jennings et al. 2001; Li and Yen 2008). Another mycotoxigenic fungus, A. flavus, was recently found to induce ET biosynthesis genes during seed infection (Wang et al. 2017). It is interesting that the induction of ZmACS6 and ZmACO3/4 by A. flavus was much greater compared with this study. This difference may be explained by the variation in timing and strategies associated with subkernel

A
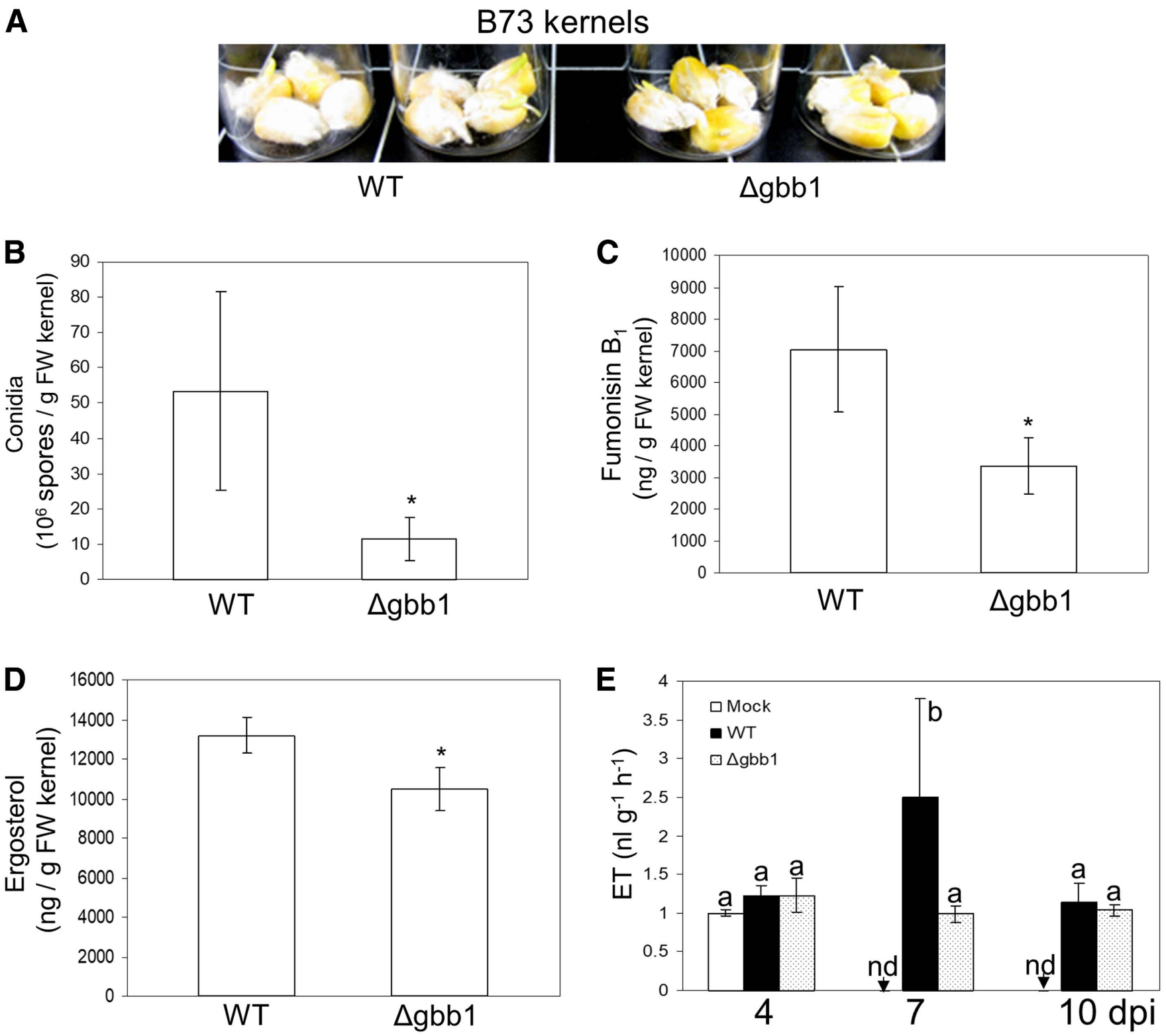

Fig. 5. Kernel infection assay in B73 kernels in response to Fusarium verticillioides and $\Delta g b b 1$ strains. A, The kernels were infected and incubated for 7 days. Phenotypes of B73 kernels infected by $F$. verticillioides wild-type (WT) and $\Delta$ gbbl were observed. B, Conidiation of F. verticillioides WT and $\triangle g b b 1$ grown on the B73 kernels was measured at 7 days. C, Fumonisin $\mathrm{B}_{1}$ was quantified at 7 days, using high performance liquid chromatography (HPLC). D, Ergosterol, a fungal-specific membrane lipid, was measured by HPLC. E, Ethylene (ET) was measured at 4, 7, and 10 days postinfection (dpi) with these two strains and control. Bars represent means of four or five biological replicates \pm standard deviation. Letters and asterisks above the bars indicate a significant difference $(P=0.05)$ between genotypes. $\mathrm{nd}=$ not detectable. 
colonization by these two fungi (Shu et al. 2017). A. flavus infection progresses much faster compared with $F$. verticillioides infection, and the two fungi likely kill their seed hosts after 4 and 9 dpi, respectively, as visualized by the lack of detectable host transcripts after these timepoints (Battilani et al. 2018; Christensen et al. 2012).

During $F$. verticillioides infection, ET was produced at high levels in WT seed between 4 and $7 \mathrm{dpi}$, as compared with mock-inoculated seeds (Fig. 2B). Compared with the ET levels induced by $F$. verticillioides, infections by A. flavus also generated considerable levels of ET, albeit to much higher absolute values (Wang et al. 2017). This suggests that ET production is a common response in maize seed during fungal infection. While ET production by seed is required for virulence of both $F$. verticillioides (this study) and A. flavus (Wang et al. 2017), the same acs 2 and acs6 mutants used in the two studies responded to the infections in a completely opposite manner. While both mutants were unable to produce ET in response to $F$. verticillioides (Fig. 2C), acs 2 and acs6 single mutants generated normal or even increased levels of ET compared with WT during A. flavus infection and were more susceptible to this pathogen (Wang et al. 2017). A. flavus-induced ET in acs2 and acs6 mutants is at least partially dependent on overexpression of the remaining functional ACS paralogues, especially ACS7 (Wang et al. 2017). It is not clear why the disruption of either ZmACS2 or ZmACS6 resulted in a strong reduction of $F$. verticillioides-induced ET, as these paralogues belong to distinct phylogenetic clades (Fig. 1C) and do not appear to be functionally redundant in this pathosystem.

While ET has been traditionally considered as a major phytohormone responsible for defense against necrotrophic pathogens, recent evidence suggests its role in promoting pathogen virulence, especially diseases caused by Fusarium spp. (Di et al. 2016). Current research and studies by Wang et al. (2017) support the notion that the role of ET in plant defense is strongly dependent on the pathosystem being evaluated. Arabidopsis plants with disrupted functions of ET signal transduction components ETR1, EIN2, and EIN3 were more resistant to $F$. graminearum, while ET overproducers eto1, eto2, and constitutive ET-activated mutant ctrl were more susceptible (Chen et al. 2009; Yelli et al. 2018). Also, wheat with attenuated expression of EIN2 displayed increased resistance to colonization by $F$. graminearum, accompanied by reduced levels of mycotoxin deoxynivalenol (Chen et al. 2009). Similar to F. graminearum, disruption of the ET receptor ETR1 made Arabidopsis more resistant to $F$. oxysporum (Pantelides et al. 2013). Unfortunately, the majority of these studies did not quantify ET emissions during infection, which likely limited the interpretation of those

A

B73 kernels
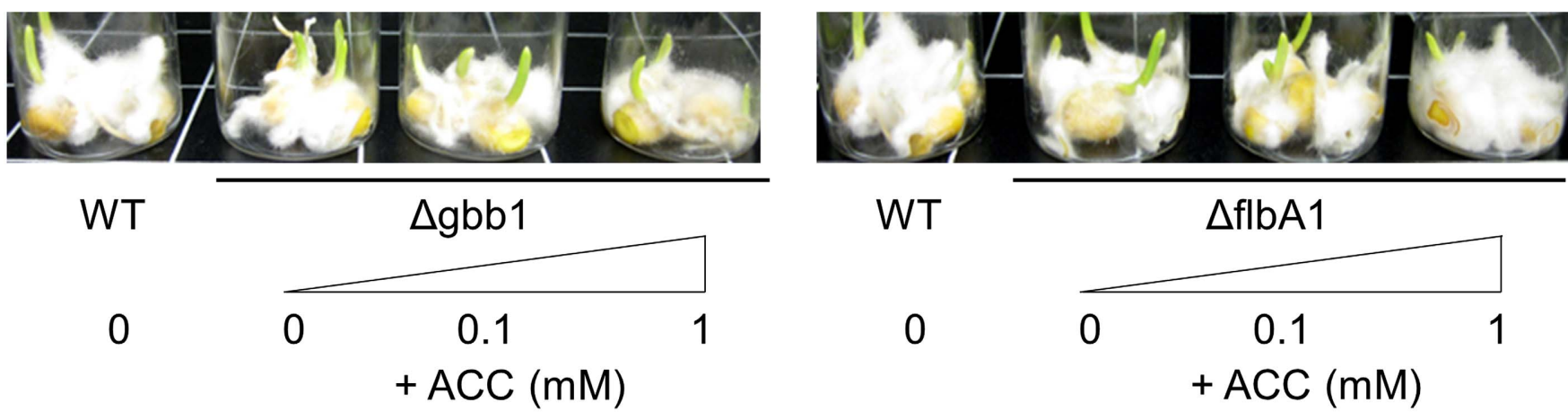

B

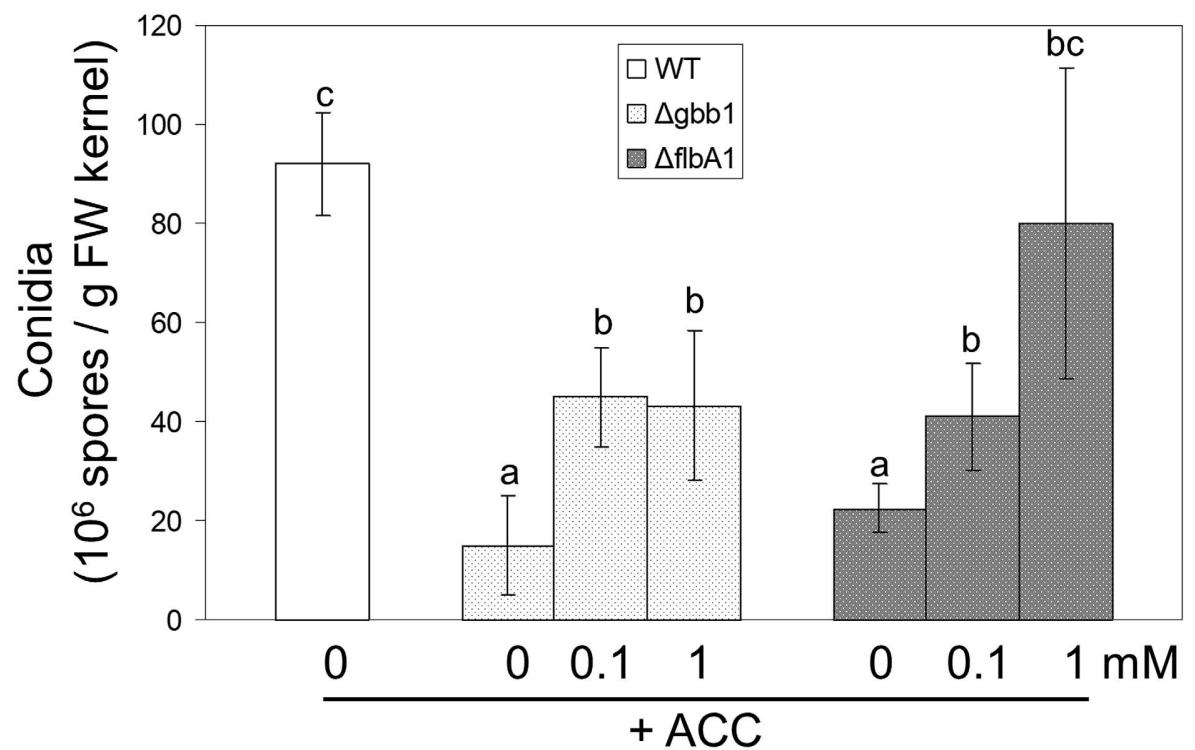

Fig. 6. 1-Aminocyclopropane-1-carboxylate (ACC), a precursor of ethylene (ET), rescues pathogenicity of $\Delta$ gbbl and $\Delta$ flbAl Fusarium verticillioides strains on maize seed. A, Effect of ACC treatment in the regulation of colonization was evaluated on B73 kernels infected with wild-type (WT), $\Delta g b b 1$, and $\Delta f l b A 1$. B, Conidiaion on B73 kernels infected with WT, $\Delta g b b 1$, and $\Delta f l b A l$ was quantified by hemocytometer. The infected kernels were treated with either control or ACC $(0.1$ and $1 \mathrm{mM})$ for 9 days at $27^{\circ} \mathrm{C}$. Letters above the bars indicate significant difference $(P=0.05)$ between genotypes by analysis of variance. 
results. For example, maize acs 2 acs6 double mutant roots were shown to be more susceptible to $F$. graminearum (Zhou et al. 2019), however, it remains to be answered whether the mutant roots produced ET in response to infection.

This study provides genetic, biochemical, and pharmacological evidence that intact ET biosynthesis contributes to maize kernels susceptibility to $F$. verticillioides. It is important to note that the results of these kernel bioassays will need to be validated by performing plant infections of the acs mutants under field and greenhouse conditions. Also, it remains unclear as to why, while disruption of either ZmACS2 or ZmACS6 resulted in a similar fivefold reduction in colonization, disruption of ZmACS6 was more impactful in both conidiation and $\mathrm{FB}_{1}$ production. Presently, it is also unclear as to why the acs 2 acs 6 double mutants supported numerically intermediate fungal colonization.

The role of ET as a susceptibility factor in maize- $F$. verticillioides interactions contrasts with that of jasmonic acid (JA), which is required for defense against $F$. verticillioides in maize mesocotyls and seed (Battilani et al. 2018; Christensen et al. 2014). Although ET and JA are typically considered to be synergistic in regulating many biological processes (He et al. 2017), there are known instances where ET and JA antagonize each other under specific stresses (Zhu and Lee 2015). In the future, maize $-F$. verticillioides interactions may prove a useful system to dissect the antagonism of these two hormones during maize responses to biotic stresses, by using a both JA- and ET-deficient mutant.

Another major finding of this study is that ET biosynthesis in the host seed may be manipulated by engaging the G-protein signaling pathway of the fungus. We have tested the involvement of this pathway in the regulation of the ET synthesis by screening G-protein signaling mutants that we previously showed to have lost virulence on maize kernels. Remarkably, null mutations in several genes involved in G-protein signaling disrupted the ET induction in the host, and these outcomes correlated with their reduced virulence on maize seed (Mukherjee et al. 2011) (Fig. 5). Because virulence of the regulator of G-protein signaling mutant $\triangle f b A l$ and $\mathrm{G}$ protein $\beta$ subunit mutant $\Delta g b b 1$ on seed is rescued by the exogenous application of the ET precursor ACC (Fig. 6), the reduced virulence of these two fungal mutants can be explained by their inability to induce ET biosynthesis in the host. Currently, the specific mechanism of how ET promotes virulence in $F$. verticillioides is not clear. One such mechanism may involve ET-mediated regulation of fungal transcriptional reprogramming. Chagué et al. (2006) reported that Botrytis cinerea recognizes exogenous ET by engaging the G-protein signaling pathway, which results in upregulation of the genes encoding virulence factors. While searching for fungal $G$ protein-dependent signals responsible for regulating host ET, we discovered a metabolite that correlates with fungal virulence (Supplementary Fig. S6). Currently the exact structure of this molecule has not been determined and will require NMR for the identification.

Additionally, our study first provided a comprehensive bioinformatic assessment of ET biosynthetic and signaling genes in the most updated version of the maize B73 genome (Jiao et al. 2017). Compared with previous review by Yang et al. (2015), our analysis uncovered 17 previously unaccounted for maize genes in several ET biosynthetic and signaling gene families (two ACS, seven ACO, one CTR, three ETR, and two EIN3) and assigned the members into distinct clades within those families. The maize genome encodes five ET-producing ACS isoforms (ZmACS1, ZmACS2, ZmACS3, ZmACS6, and ZmACS7) compared with nine in Arabidopsis (Yamagami et al. 2003) (Fig. 1A). As many as five of the Arabidopsis ACS genes cluster with ZmACS6, and this may explain why studies utilizing ET biosynthetic mutants are lacking. Higher order Arabidopsis mutants may be required to recapitulate the phenotypes observed by the disruption of ZmACS6. Thus, maize is well positioned as an advantageous system to explore the role of ET biosynthesis in plant biology and plant-organismal interactions. In contrast to the reduced number of ACS genes in maize relative to Arabidopsis, the maize $A C O$ gene family contains 13 members and appears to have experienced several duplication events, as compared with Arabidopsis. Two groups of tandemly duplicated genes were uncovered. $\mathrm{ZmACO} 3 a$ and $\mathrm{ZmACO} 3 \mathrm{~b}$ are adjacent to each other on chromosome 2 and the entire $\mathrm{ZmACO} 4$ clade consists of four genes that are in close proximity to one another on chromosome 10. The ZmACO3a and ZmACO3b pair and the $\mathrm{ZmACO} 4 a, \mathrm{ZmACO} 4 b$, and $\mathrm{ZmACO} 4 c$ triplet are nearly identical paralogues (NIPs) and share $>98 \%$ identity with the NIP group (Emrich et al. 2007). ZmACO4 NIPs are located within a Fusarium resistance QTL identified in a study by Samayoa et al. (2019) and, thus, represents an interesting group of candidate genes for investigating their relevance for resistance or susceptibility to $F$. verticillioides.

\section{MATERIALS AND METHODS}

\section{Bioinformatics.}

The maize genome database (Cannon et al. 2011) was searched for ET-related genes, using Arabidopsis and rice orthologous amino acid sequences (Yang et al. 2015). Evolutionary analyses were conducted in MEGA X (Kumar et al. 2018), using amino acid sequence alignment by MUSCLE and a maximum likelihood method based on the JTT matrix-based model (Jones et al. 1992).

\section{Plant materials and fungal strains.}

Maize acs mutants (acs 2 and acs 6 ) were generated by Mutator-transposable element-insertional mutagenesis as described previously (Young et al. 2004). These acs mutants were backcrossed seven times into the B73 inbred line, producing mutant lines that were near-isogenic to the recurrent parent line B73.

$F$. verticillioides 7600 and $F$. verticillioides $\Delta g b b 1$ and $\triangle f b A 1$ mutants were cultured at room temperature on potato dextrose agar (PDA) (Difco), as described previously (Gao et al. 2007; Mukherjee et al. 2011; Sagaram and Shim 2007).

\section{Fungal inoculation and spore counting on kernels.}

Maize kernel infections were performed according to Christensen et al. (2012). Maize kernels of similar size were particularly selected and were weighed to ensure equivalent average seed weights across all tested samples. Seeds were then surfacesterilized with $6 \%$ sodium hypochlorite for 10 to $15 \mathrm{~min}$ and were rinsed with sterilized distilled water (SDW) at least five times. The embryos of kernels were cut longitudinally, using a razor blade, to a depth of about $0.5 \mathrm{~cm}$, to provide an infection count for fungal inoculation. Seeds were then dried with a paper towel, were placed in a $20-\mathrm{ml}$ glass-scintillation vial (Wheaton Science), and were inoculated with $200 \mu \mathrm{l}$ of conidia suspension ( $10^{6}$ spores per milliliter) of $F$. verticillioides $7600, \Delta g b b 1$, and $\triangle f b A l$ strains. Conidia were harvested with $0.001 \%$ Tween-20 from fungal strains grown on a PDA plate. Control seeds (mock) received an equal volume of $0.001 \%$ Tween-20. Four inoculated or mock-treated kernels were used per replicate with at least four replicates per treatment or genotype for all experiments. The inoculated kernels were kept in a plastic transparent container, with a wet filter paper to provide humidity, and were incubated with a 12-h light and dark cycle at $27^{\circ} \mathrm{C}$. SDW was added to containers as needed to maintain high humidity for fungal growth. Kernels were harvested at designated intervals after inoculation, either to enumerate conidia or to quantify $\mathrm{FB}_{1}$. 
To measure levels of conidia production, infected kernels were placed in a $20-\mathrm{ml}$ glass vial with $2 \mathrm{ml}$ of $0.001 \%$ Tween20 and were vortexed for $20 \mathrm{~s}$ to dislodge spores. The spore suspension was decanted, and spores were enumerated using a hemocytometer under an optical microscope.

\section{Total RNA extraction, complementary DNA (cDNA) synthesis, and semiquantitative reverse transcription (RT)-PCR.}

B73 inbred line kernels were surface-sterilized and were inoculated, with either mock control or the WT strain of $F$. verticillioides, as described above. Noninoculated control or inoculated kernels were harvested at $0,12,24,48$, and $96 \mathrm{~h}$ after treatment. Total RNA was extracted using TRI reagent (Molecular Research Center Inc.) following manufacturer protocol. RNA samples were treated with RNase-free $\mathrm{rDNase}$ at $37^{\circ} \mathrm{C}$ for 30 min, using a DNA-free kit (Ambion Inc.). First strand cDNA synthesis ( $5 \mu \mathrm{g}$ of RNA as a template for each sample) was carried out using a First-Strand synthesis kit (GE Healthcare BioSciences Corp.), following manufacturer protocol. Synthesized cDNA was diluted and equalized, using glycerol phosphate dehydrogenase $(Z m G A P c)$ as a housekeeping gene for all samples. cDNA was used as a template for amplification with two gene-specific primers for each gene. cDNA was denatured at $94^{\circ} \mathrm{C}$ for $5 \mathrm{~min}$ and was amplified by following with 27 to 32 cycles (each cycle: $45 \mathrm{~s}$ at $94^{\circ} \mathrm{C}, 1 \mathrm{~min}$ at $56^{\circ} \mathrm{C}$, and $2 \mathrm{~min}$ at $72^{\circ} \mathrm{C}$ ). Amplified PCR products were loaded and separated on $1.5 \%$ agarose gels. Primers for RT-PCR in this study are listed in Supplementary Table S2.

\section{Quantification of $\mathbf{F B}_{\mathbf{1}}$ and ergosterol.}

$\mathrm{FB}_{1}$ measurement was conducted using high performance liquid chromatography (HPLC) as described in the kernel bioassay method paper by Christensen et al. (2012). Four infected kernels per replicate were frozen in liquid nitrogen until assayed. Five replicates were used for $\mathrm{FB}_{1}$ measurement. Mycotoxin concentrations in nanograms per gram were log-transformed to equalize variance. Data were subjected to an analysis of variance and Fisher's protected least significant difference test was used to separate means.

Ergosterol was extracted from infected kernels overnight with $5 \mathrm{ml}$ of chloroform/methanol $(2: 1, \mathrm{vol} / \mathrm{vol})$ at room temperature as described previously (Woloshuk et al. 1979), with some modifications. Ergosterol was quantified on a Shimatzu LC-20AT HPLC system (Shimatzu Scientific Instruments, Inc.) equipped with a $4.6 \mathrm{U}$ ODS column $(250 \times 4.6 \mathrm{~mm})$ and a UV detector (282 nm). Quantities were calculated by comparing HPLC peak areas with ergosterol standards (Sigma). All experiments were repeated at least two times, with consistent results.

\section{Measurement of endogenous ET produced by maize kernels and pathogens.}

ET produced by noninoculated and $F$. verticillioides-inoculated kernels was quantified as described previously (Gao et al. 2008), with some modifications. Briefly, the vials containing infected kernels were kept with a 12-h light and dark cycle at $27^{\circ} \mathrm{C}$, and ET was measured at 1, 2, 4, and 7 dpi. Vials were sealed with screw caps with septa. One milliliter of headspace gas was withdrawn from vials by syringe and was analyzed, using a digital gas chromatography (Photovac 10 plus; PerkinElmer, Inc.) with a photo detector and compressed air (ultrazero grade; Praxair, Inc.) as carrier gas.

\section{Statistical analysis.}

Analysis of variance of experimental datasets was performed using JMP software version 5.0 (SAS Institute Inc.). Significant effects of treatment were determined by the magnitude of the
F-value $(P=0.05)$. When a significant F-test was obtained, separation of means was accomplished by Fisher's protected least significant difference at $P=0.05$.

\section{Distribution of materials.}

Novel materials described in this publication may be available for noncommercial research purposes upon acceptance and signing of a material transfer agreement. In some cases, such materials may contain or be derived from materials obtained from a third party. In such cases, distribution of material will be subject to the requisite permission from any third-party owners, licensors, or controllers of all or parts of the material. Obtaining any permission will be the sole responsibility of the requestor. Plant germplasm will not be made available except at the discretion of the owner and then only in accordance with all applicable governmental regulations.

\section{ACKNOWLEDGMENTS}

We thank D. R. Gallie (University of California-Riverside) for his kind gift of the seed of the acs 2 and acs 6 mutants used in this study.

\section{LITERATURE CITED}

Adie, B., Chico, J. M., Rubio-Somoza, I., and Solano, R. 2007. Modulation of plant defenses by ethylene. J. Plant Growth Regul. 26:160-177.

Bari, R., and Jones, J. D. G. 2009. Role of plant hormones in plant defence responses. Plant Mol. Biol. 69:473-488.

Barkai-Golan, R., Lavy-Meir, G., and Kopeliovitch, E. 1989. Effects of ethylene on the susceptibility to Botrytis cinerea infection of different tomato genotypes. Ann. Appl. Biol. 114:391-396.

Battilani, P., Lanubile, A., Scala, V., Reverberi, M., Gregori, R., Falavigna, C., Dall'asta, C., Park, Y. S., Bennett, J., Borrego, E. J., and Kolomiets, M. V. 2018. Oxylipins from both pathogen and host antagonize jasmonic acid-mediated defence via the 9-lipoxygenase pathway in Fusarium verticillioides infection of maize. Mol. Plant Pathol. 19:2162-2176.

Broekaert, W. F., Delauré, S. L., De Bolle, M. F. C., and Cammue, B. P. 2006. The role of ethylene in host-pathogen interactions. Annu. Rev. Phytopathol. 44:393-416.

Cannon, E. K., Birkett, S. M., Braun, B. L., Kodavali, S., Jennewein, D. M., Yilmaz, A., Antonescu, V., Antonescu, C., Harper, L. C., Gardiner, J. M., Schaeffer, M. L., Campbell, D. A., Andorf, C. M., Andorf, D., Lisch, D., Koch, K. E., McCarty, D. R., Quackenbush, J., Grotewold, E., Lushbough, C. M., Sen, T. Z., and Lawrence, C. J. 2011. POPcorn: An online resource providing access to distributed and diverse maize project data. Int. J. Plant Genomics 2011:923035.

Chagué, V., Danit, L. V., Siewers, V., Schulze-Gronover, C., Tudzynski, P., Tudzynski, B., and Sharon, A. 2006. Ethylene sensing and gene activation in Botrytis cinerea: A missing link in ethylene regulation of fungus-plant interactions? Mol. Plant-Microbe Interact. 19:33-42.

Chen, X., Steed, A., Travella, S., Keller, B., and Nicholson, P. 2009. Fusarium graminearum exploits ethylene signalling to colonize dicotyledonous and monocotyledonous plants. New Phytol. 182:975-983.

Christensen, S., Borrego, E., Shim, W. B., Isakeit, T., and Kolomiets, M. 2012. Quantification of fungal colonization, sporogenesis, and production of mycotoxins using kernel bioassays. J. Vis. Exp. 23:3727.

Christensen, S. A., Nemchenko, A., Park, Y. S., Borrego, E., Huang, P. C., Schmelz, E. A., Kunze, S., Feussner, I., Yalpani, N., Meeley, R., and Kolomiets, M. V. 2014. The novel monocot-specific 9-lipoxygenase ZmLOX12 is required to mount an effective jasmonate-mediated defense against Fusarium verticillioides in maize. Mol. Plant-Microbe Interact. 27:1263-1276.

Cristescu, S. M., De Martinis, D., Te Lintel Hekkert, S., Parker, D. H., and Harren, F. J. 2002. Ethylene production by Botrytis cinerea in vitro and in tomatoes. Appl. Environ. Microbiol. 68:5342-5350.

Di, X., Takken, F. L., and Tintor, N. 2016. How phytohormones shape interactions between plants and the soil-borne fungus Fusarium oxysporum. Front. Plant Sci. 7:170.

Ding, X., Cao, Y., Huang, L., Zhao, J., Xu, C., Li, X., and Wang, S. 2008. Activation of the indole-3-acetic acid-amido synthetase GH3 8 suppresses expansin and promotes salicylate- and jasmonateindependent basal immunity in rice. Plant Cell 20:228-240. 
El-Kazzaz, M. K., Sommer, N. F., and Kader, A. A. 1983. Ethylene effects on in vitro and in vivo growth of certain postharvest fruitinfecting fungi. Phytopathology 73:998-1001.

Ellis, C., and Turner, J. G. 2001. The Arabidopsis mutant cev1 has constitutively active jasmonate and ethylene signal pathways and enhanced resistance to pathogens. Plant Cell 13:1025-1033.

Emrich, S. J., Li, L., Wen, T. J., Yandeau-Nelson, M. D., Fu, Y., Guo, L., Chou, H. H., Aluru, S., Ashlock, D. A., and Schnable, P. S. 2007. Nearly identical paralogs: Implications for maize (Zea mays L.) genome evolution. Genetics 175:429-439.

Gane, R. 1934. Production of ethylene by some ripening fruits. Nature $134: 1008$.

Gao, X., Shim, W. B., Göbel, C., Kunze, S., Feussner, I., Meeley, R., Balint-Kurti, P., and Kolomiets, M. 2007. Disruption of a maize 9-lipoxygenase results in increased resistance to fungal pathogens and reduced levels of contamination with mycotoxin fumonisin. Mol. Plant-Microbe Interact. 20:922-933.

Gao, X., Starr, J., Göbel, C., Engelberth, J., Feussner, I., Tumlinson, J., and Kolomiets, M. 2008. Maize 9-lipoxygenase ZmLOX3 controls development, root-specific expression of defense genes, and resistance to root-knot nematodes. Mol. Plant-Microbe Interact. 21:98-109.

Glazebrook, J., Chen, W., Estes, B., Chang, H. S., Nawrath, C., Métraux, J. P., Zhu, T., and Katagiri, F. 2003. Topology of the network integrating salicylate and jasmonate signal transduction derived from global expression phenotyping. Plant J. 34:217-228.

Gottwald, S., Samans, B., Lück, S., and Friedt, W. 2012. Jasmonate and ethylene dependent defence gene expression and suppression of fungal virulence factors: Two essential mechanisms of Fusarium head blight resistance in wheat? BMC Genomics 13:369.

Guo, H., and Ecker, J. R. 2004. The ethylene signaling pathway: New insights. Curr. Opin. Plant Biol. 7:40-49.

He, X., Jiang, J., Wang, C. Q., and Dehesh, K. 2017. ORA59 and EIN3 interaction couples jasmonate-ethylene synergistic action to antagonistic salicylic acid regulation of PDF expression. J. Integr. Plant Biol. 59:275287.

Jennings, J. C., Apel-Birkhold, P. C., Mock, N. M., Baker, C. J., Anderson, J. D., Bailey, and B. A. 2001. Induction of defense responses in tobacco by the protein Nep1 from Fusarium oxysporum. Plant Sci. 161:891-899.

Jiao, Y., Peluso, P., Shi, J., Liang, T., Stitzer, M. C., Wang, B., Campbell, M. S., Stein, J. C., Wei, X., Chin, C. S., Guill, K., Regulski, M., Kumari, S., Olson, A., Gent, J., Schneider, K. L., Wolfgruber, T. K., May, M. R., Springer, N. M., Antoniou, E., McCombie, W. R., Presting, G. G., McMullen, M., Ross-Ibarra, J., Dawe, R. K., Hastie, A., Rank, D. R., and Ware, D. 2017. Improved maize reference genome with single-molecule technologies. Nature 546:524-527.

Johnson, P. R., and Ecker, J. R. 1998. The ethylene gas signal transduction pathway: A molecular perspective. Annu. Rev. Genet. 32:227-254.

Jones, D. T., Taylor, W. R., and Thornton, J. M. 1992. The rapid generation of mutation data matrices from protein sequences. Comput. Appl. Biosci. 8:275-282.

Kende, H. 1993. Ethylene biosynthesis. Annu. Rev. Plant Physiol. Plant Mol. Biol. 44:283-307.

Kendrick, M. D., and Chang, C. 2008. Ethylene signaling: New levels of complexity and regulation. Curr. Opin. Plant Biol. 11:479-485.

Kumar, S., Stecher, G., Li, M., Knyaz, C., and Tamura, K. 2018. MEGA $\mathrm{X}$ : Molecular evolutionary genetics analysis across computing platforms. Mol. Biol. Evol. 35:1547-1549.

Kunkel, B. N., and Brooks, D. M. 2002. Cross talk between signaling pathways in pathogen defense. Curr. Opin. Plant Biol. 5:325-331.

Lanubile, A., Maschietto, V., Borrelli, V. M., Stagnati, L., Logrieco, A. F., and Marocco, A. 2017. Molecular basis of resistance to Fusarium ear rot in maize. Front. Plant Sci. 8:1774.

Li, G., and Yen, Y. 2008. Jasmonate and ethylene signaling pathway mediate fusarium head blight resistance in wheat. Crop Sci. 48:1888-1896.

Lin, Z., Zhong, S., and Grierson, D. 2009. Recent advances in ethylene research. J. Exp. Bot. 60:3311-3336.
Ma, K. W., and Ma, W. 2016. Phytohormone pathways as targets of pathogens to facilitate infection. Plant Mol. Biol. 91:713-725.

Maschietto, V., Colombi, C., Pirona, R., Pea, G., Strozzi, F., Marocco, A., Rossini, L., and Lanubile, A. 2017. QTL mapping and candidate genes for resistance to Fusarium ear rot and fumonisin contamination in maize. BMC Plant Biol. 17:20.

Mukherjee, M., Kim, J. E., Park, Y. S., Kolomiets, M. V., and Shim, W. B. 2011. Regulators of G-protein signalling in Fusarium verticillioides mediate differential host-pathogen responses on nonviable versus viable maize kernels. Mol. Plant Pathol. 12:479-491.

Munkvold, G. P. 2003. Cultural and genetic approaches to managing mycotoxins in maize. Annu. Rev. Phytopathol. 41:99-116.

Pantelides, I. S., Tjamos, S. E., Pappa, S., Kargakis, M., and Paplomatas, E. J. 2013. The ethylene receptor ETR 1 is required for Fusarium oxysporum pathogenicity. Plant Pathol. 62:1302-1309.

Penninckx, I. A., Thomma, B. P., Buchala, A., Métraux, J. P., and Broekaert, W. F. 1998. Concomitant activation of jasmonate and ethylene response pathways is required for induction of a plant defensin gene in Arabidopsis. Plant Cell 10:2103-2113.

Robertson Hoyt, L. A., Jines, M. P., Balint-Kurti, P., Kleinschmidt, C. E., White, D. G., Payne, G. A., Maragos, C. M., Molnar, T. L., and Holland, J. B. 2006. QTL mapping for Fusarium ear rot and fumonisin contamination resistance in two maize populations. Crop Sci. 46:1734-1743.

Ruduś, I., Sasiak, M., and Kepczynski, J. 2013. Regulation of ethylene biosynthesis at the level of 1-aminocyclopropane-1-carboxylate oxidase (ACO) gene. Acta Physiol. Plant. 35:295-307.

Sagaram, U. S., and Shim, W. B. 2007. Fusarium verticillioides GBB1, a gene encoding heterotrimeric $G$ protein $\beta$ subunit, is associated with fumonisin $\mathrm{B}_{1}$ biosynthesis and hyphal development but not with fungal virulence. Mol. Plant Pathol. 8:375-384.

Samayoa, L. F., Cao, A., Santiago, R., Malvar, R. A., and Butrón, A. 2019. Genome-wide association analysis for fumonisin content in maize kernels. BMC Plant Biol. 19:166.

Shu, X., Livingston, D. P., 3rd, Woloshuk, C. P., and Payne, G. A. 2017. Comparative histological and transcriptional analysis of maize kernels infected with Aspergillus flavus and Fusarium verticillioides. Front. Plant Sci. 8:2075.

Wang, S., Park, Y. S., Yang, Y., Borrego, E. J., Isakeit, T., Gao, X., and Kolomiets, M. V. 2017. Seed-derived ethylene facilitates colonization but not aflatoxin production by Aspergillus flavus in maize. Front. Plant Sci. 8:415.

Woloshuk, C. P., Sisler, H. D., and Dutky, S. R. 1979. Mode of action of the azasteroid antibiotic 15-aza-24 methylene-d-homocholesta-8,14-dien-3 beta-ol in Ustilago maydis. Antimicrob. Agents Chemother. 16:81-97.

Yamagami, T., Tsuchisaka, A., Yamada, K., Haddon, W. F., Harden, L. A., and Theologis, A. 2003. Biochemical diversity among the 1amino-cyclopropane-1-carboxylate synthase isozymes encoded by the Arabidopsis gene family. J. Biol. Chem. 278:49102-49112.

Yang, C., Lu, X., Ma, B., Chen, S. Y., and Zhang, J. S. 2015. Ethylene signaling in rice and Arabidopsis: Conserved and diverged aspects. Mol. Plant 8:495-505.

Yang, S. F., and Hoffman, N. E. 1984. Ethylene biosynthesis and its regulation in higher-plants. Annu. Rev. Plant Physiol. Plant Mol. Biol. 35:155-189.

Yelli, F., Kato, T., and Nishiuchi, T. 2018. The possible roles of AtERF71 in the defense response against the Fusarium graminearum. Plant Biotechnol Tokyo 35:187-192.

Young, T. E., Meeley, R. B., and Gallie, D. R. 2004. ACC synthase expression regulates leaf performance and drought tolerance in maize. Plant J. 40:813-825.

Zhou, S., Zhang, Y. K., Kremling, K. A., Ding, Y., Bennett, J. S., Bae, J. S., Kim, D. K., Ackerman, H. H., Kolomiets, M. V., Schmelz, E. A., Schroeder, F. C., Buckler, E. S., and Jander, G. 2019. Ethylene signaling regulates natural variation in the abundance of antifungal acetylated diferuloylsucroses and Fusarium graminearum resistance in maize seedling roots. New Phytol. 221:2096-2111.

Zhu, Z., and Lee, B. 2015. Friends or foes: New insights in jasmonate and ethylene co-actions. Plant Cell Physiol. 56:414-420. 